\title{
Unsteady Viscous Dissipative Flow of Salty Water in the Presence of a Magnetic Field in a Semi-Infinite Porous Medium
}

\author{
Ronald Nyabutoํㅜ, Rogers Omboga Amenya², Jeconniah Abonyo Okelo ${ }^{1}$ \\ ${ }^{1}$ Department of Pure and Applied Mathematics, Jomo Kenyatta University of Agriculture and Technology, Juja, Kenya \\ ${ }^{2}$ Department of Mathematical and Physical Sciences, Dedan Kimathi University of Technology, Nyeri, Kenya \\ Email: romboga2010@gmail.com
}

How to cite this paper: Nyabuto, R., Amenya, R.O. and Okelo, J.A. (2021) Unsteady Viscous Dissipative Flow of Salty Water in the Presence of a Magnetic Field in a Semi-Infinite Porous Medium. Journal of Applied Mathematics and Physics, 9, 931-940.

https://doi.org/10.4236/jamp.2021.95063

Received: March 9, 2021

Accepted: May 16, 2021

Published: May 19, 2021

Copyright $\odot 2021$ by author(s) and Scientific Research Publishing Inc. This work is licensed under the Creative Commons Attribution International License (CC BY 4.0).

http://creativecommons.org/licenses/by/4.0/

\begin{abstract}
In this paper, an investigation of unsteady free convective magneto-hydro dynamic (MHD) flow of salty water in the presence of a transverse magnetic field has been carried out. An electrically conducting and incompressible fluid is assumed to flow past a semi-infinite vertical porous plate. A constant magnetic field is applied normally to the vertical plate. The Prandtl numbers for salt water are taken to be 1 at $20^{\circ} \mathrm{C}$. The governing equations of the problem contain non-linear partial differential equations that are transformed into ordinary differential equations. An explicit finite difference scheme has been used to solve the transformed ordinary equations generated from this flow problem. The velocity profiles and temperature distribution of the flow field have been discussed and presented graphically.
\end{abstract}

\section{Keywords}

Viscous Dissipative, Porous Medium, Unsteady

\section{Introduction}

Water is a very useful fluid in every day to day operations for engineers and generally in life. Water can be pure or impure. In this study, pure water refers to fresh water, while impure water is water containing impurities. Impurities are foreign materials introduced to fresh water. These materials include dust, oil smoke salts among others.

In engineering, fresh and salt water have contributed immensely to the advancement of scientific innovations of machines such ships and generation of electricity. It is noted that in this research, salty water constantly interacts with 
the earth's magnetic field and thus becomes electrically conducting. The science that involves motion of an electrically conducting fluid over a surface in the presence of a magnetic field is known as magneto-hydro dynamics (MHD). The fluids that are commonly encountered include liquid metals, such as mercury, molten magnesium, liquid sodium, gallium etc. and plasmas such as salty water or electrolyte and solar atmosphere. The influence of the magnetic field on an electrically conducting fluid is important in many scientific fields, such as in purification of crude oil, in design of MHD generators, pumps and accelerators among others. Further, engineers employ the principles of MHD in the design of heat exchangers, in the design of communications and radar systems and in creating power generating systems. In this research, our focus dwelt on salty water due to its abundance in lakes, oceans and seas. The design of high-speed boats that utilize MHD flow of salty water and MHD generator is part of the technological advancements that has led to the growth of science today.

\section{Literature Review}

The encounter of a magnetic field in the presence of salty water is not a new occurrence. With this fact, a number of studies have been made on MHD and the contribution of magnetic field in flows of salty water. Considerable progress on modifications and improvements on designs and manufacture of scientific machines and equipments has been made. Among the recent studies made on this topic includes a research done by Scholars [1]. They conducted a study on heat and mass transfer along a semi-infinite vertical flat plate under the combined buoyancy force effects of thermal and species diffusion in the presence of a strong non-uniform magnetic field. The researchers used a fourth-order RungeKutta scheme with the shooting method to solve numerically their similarity equations. On the other hand, [2] made a research on Heat transfer and MHD flow of non-Newtonian Maxwell fluid through a parallel plate channel. They used fourth order RungeKutta numerical method to solve their governing equations and found that an increase in Hartmann number cause a decrease in velocity although its increase caused an increase in temperature. Scholars in [3] carried out a study on an analysis of unsteady MHD fluid flow in a vertical semi-infinite plate with constant transverse magnetic field. They found that an increase in Hartmann number caused an increase in velocity profiles while a rise in Grashof number resulted into an increase in temperature of the flow field.

Researchers in [4] studied viscous fluid flow past a hot vertical porous plate. In this study the suction velocity was regarded constant and the wall temperature assumed spanwise cosinusoidal. The flow parameters in this study were analyzed and the solutions for the velocity, the temperature, skin friction and rate of heat transfer were obtained using perturbation method. It was observed that both the velocity and the skin friction diminished as the Magnetic parameter increased. In addition, it was realized that an increase in suction caused an increase in both velocity and the skin friction. An analysis of the MHD boundary 
layer flow over a nonlinear stretching sheet in a porous medium using semianalytical approaches was done by [5]. They found that temperature and concentration profiles decreased with increase in values of Prandtl and Schimidt numbers respectively. Further, [6] investigated on unsteady natural convective flow over an impulsively started semi-infinite vertical plate in the presence of porous medium with chemical reaction. An investigation on numerical analysis of two dimensional unsteady MHD free convection and mass transfer of fresh and salty water on an inclined plate with hall current and constant heat flux was done by [7] while Magnet-Hydro Dynamic generation was conducted by [8]. They passed salt water in the MHD generator through a duct and found that voltage was induced. Researchers in [9] carried out a study on magneto Hydrodynamics power generation using salty water. The researchers intended to develop an MHD generator that uses salty water as an electrically conducting fluid. An electrical unsteady MHD natural convection flow of Nano fluid with thermal stratisfication and heat generation/Absorption was conducted by [10] whilst researchers in [11] investigated the unsteady two-dimensional laminar flow of a viscous incompressible electrically conducting fluid in the vicinity of semi-infinite vertical porous moving plate in the presence of a transverse magnetic field. The plate moves with constant velocity in the direction of fluid flow, and the free stream velocity follows the exponentially increasing small permutation law, the effect of increasing values of the suction velocity parameter results into a slight increase in surface skin friction for lower values of plate moving velocity. It was also observed that for several values of Prandtl number, the surface heat transfer decreases by increasing the magnitude of suction velocity.

In view of the researches highlighted this research is aimed at establishing how the salt water affect the design of vessels such MHD generators and boats and ships that use salty water for routine operations. In addition, an explicit finite difference technique is employed to determine the results of the flow problem, a technique that has not been well utilized in the above citations.

\section{Formulation of the Problem}

An unsteady free convection flow of an electrically conducting, viscous, incompressible fluid past a vertical infinite porous plate is considered. In this flow problem salty water is allowed to flow though semi-infinite plates. A uniform magnetic field $\mathbf{B}$ is applied perpendicular to the plates in the positive $y$-direction. The $\mathrm{x}$-axis is taken along the infinite vertical porous plate in the upward direction and $y$-axis normal to the plate as shown in Figure 1.

In this flow problem, the plate was assumed to be porous, electrically non-conducting and very long in the $\mathrm{x}-\mathrm{z}$ direction. In addition, the salty water was regarded to be incompressible with constant density and small Reynolds number. Effects of Hall current were ignored since a weak magnetic field was applied hence generalized Ohm's law negligible.

The governing equations of the problem are expressed as follow; 


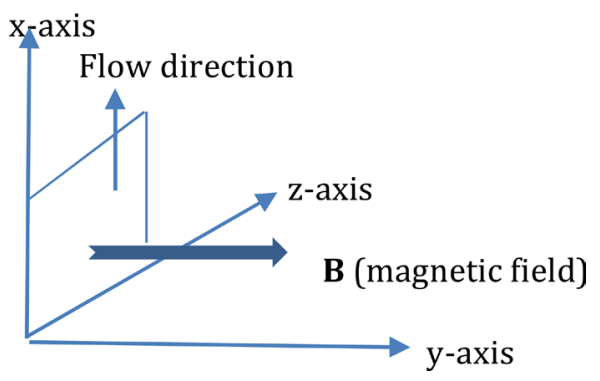

Figure 1. Configuration of flow problem.

1) The continuity equation

The equation is based on conservation of mass. The law assumes that mass is neither created nor destroyed but is transformed from one form to another. Amenya et al. (2018) gave the equation as

$$
\frac{\partial \rho}{\partial t}+(\nabla \cdot \rho v)=0
$$

In this paper, the flow is assumed to be incompressible. Thus, Equation (1) decomposes to

$$
\frac{\partial v}{\partial y}=0
$$

Integrating the Equation (2) gives

$$
V=-v_{0}
$$

The quantity $-v_{0}$ in (3) above is the suction velocity at the wall of the vertical plate.

2) The momentum equation

The equation of momentum is derived from Newton's second law of motion which states that the sum of the resultant forces is equal to the rate of change of momentum of the flow.

$$
\frac{\partial q}{\partial t}+(q \cdot \nabla) q=-\frac{1}{\rho} \nabla p+\vartheta \nabla^{2} q+F
$$

where $t$ is time, $q$ is velocity $\boldsymbol{p}$ is pressure $\rho$ is density of the fluid and $F=\boldsymbol{J} \times \boldsymbol{B}$ is the body force called Lorentz force.

The equation of continuity coupled with the simplified momentum equation result into an equation of the form

$$
\rho\left(-v_{0} \frac{\partial u}{\partial y}\right)=-\frac{\partial p}{\partial x}+\mu \frac{\partial^{2} u}{\partial y^{2}}-\rho g+\boldsymbol{J} \times \boldsymbol{B}
$$

3) Energy equation

This equation is fundamentally established from the first law of thermodynamics. Scholars in [12] express the energy equation as

$$
\rho C_{p} \frac{D_{T}}{D_{t}}=\nabla \cdot(K \nabla \cdot T)+\frac{1}{\sigma} j^{2}+\mu \varphi
$$


where $\frac{D_{T}}{D_{t}}$ is the material derivative, $C_{p}$ is the specific heat capacity at constant pressure and $\mu \varphi$ is the internal heating due to the viscous dissipation. Upholding the considerations made in this problem, the Equation (6) simplifies to

$$
\rho C_{p}\left(\frac{\partial T}{\partial t}\right)=k \frac{\partial^{2} T}{\partial y^{2}}+\mu\left(\frac{\partial u}{\partial y}\right)^{2}+\sigma B_{0}^{2} U^{2}
$$

The initial conditions are

$$
\begin{aligned}
& t \leq 0, u=0, T=0 \text { at } y=0 \\
& t>0, u=0 \text { and } T=T_{0} \quad \forall y
\end{aligned}
$$

\section{Non-Dimensionilisation}

This is the process that aims at reducing the complexity of the differential equations by selecting characteristic dimensionless quantities and then substituting the in the equations. For this work the following non-dimensional variables are used in momentum and energy equations so as to reduce their complexity

$$
\begin{aligned}
& t=t^{*} \frac{U^{2}}{\vartheta}, y=y^{*} \frac{U}{\vartheta}, \theta=\frac{T^{*}-T_{\infty}^{*}}{T_{0}^{*}-T_{\infty}^{*}}, P_{r}=\frac{C_{p} \mu}{k} \\
& u=\frac{u^{*}}{U}, v_{0}=\frac{v_{0}^{*}}{U}, E_{c}=\frac{U^{2}}{C_{p}\left(T_{w}^{*}-T_{\infty}^{*}\right)}, x^{*}=\frac{x}{l}
\end{aligned}
$$

Consequently, Equation (5) is reduced into

$$
\frac{\partial u}{\partial t}=\frac{\partial^{2} u}{\partial y^{2}}+v_{0} \frac{\partial u}{\partial y}+G r \theta
$$

On the other hand, Equation (7) collapses to

$$
\frac{\partial \theta}{\partial t}=\frac{1}{\operatorname{Pr}} \frac{\partial^{2} \theta}{\partial y^{2}}+E c\left(\frac{\partial u}{\partial y}\right)^{2}
$$

The boundary conditions applied in this flow problem are;

$$
\begin{aligned}
& t \leq 0, u=1, \theta=1 \text { at } y=0 \\
& t>0, u=0, \theta=0 \text { for all } y
\end{aligned}
$$

\section{Method of Solution}

The non-dimensionalized partial differential equations for this flow problem are solved by explicit finite difference scheme. When explicit finite difference scheme is to be used, a discrete mesh is employed. A discrete mesh is a set of locations in which finite difference equations are computed. The region of flow is divided into a grid or meshes of parallel lines to $y$ - and $t$-planes forming an image that resembles a net or a mesh as shown in Figure 2.

Thus the non-dimensionilized differential Equations (9) and (10) together with initial and boundary conditions (8) and (11) are solved numerically using an explicit the finite difference scheme. In this scheme, a differential equation is 

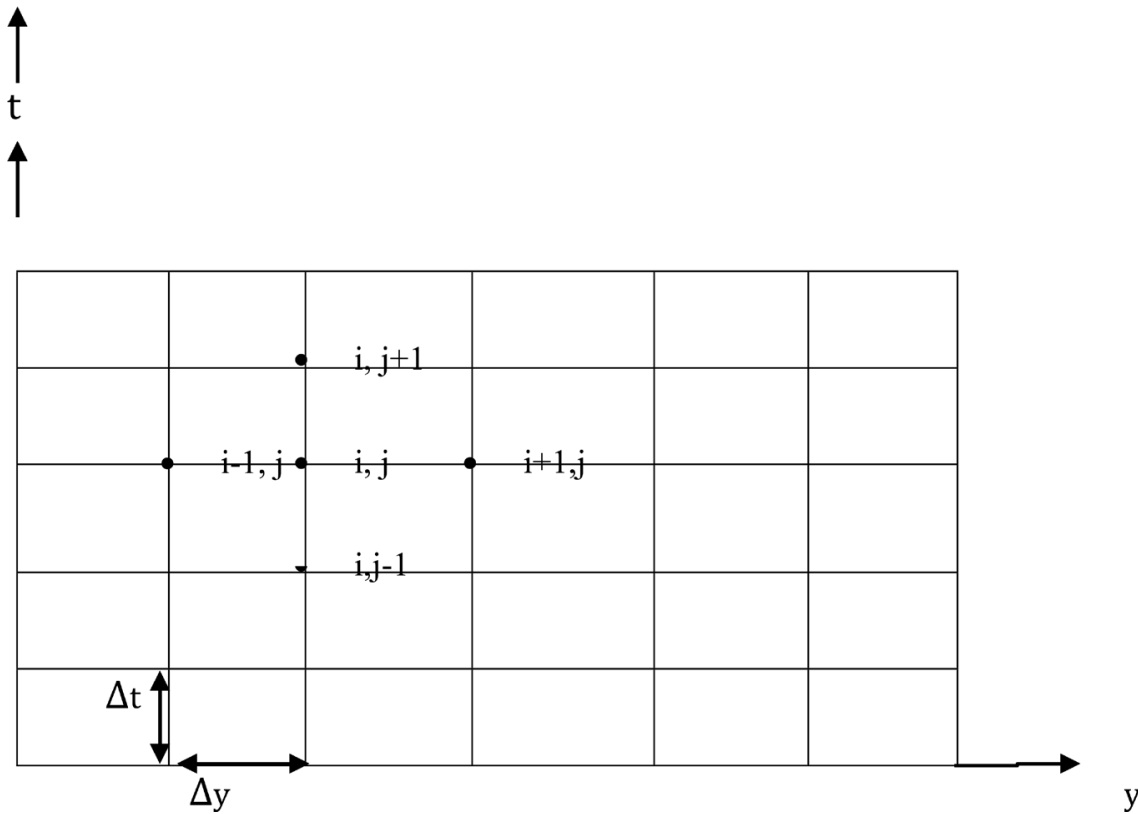

Figure 2. Uniform mesh.

replaced with a discrete approximation which yield into a set of algebraic equations that are solved for the values of the discrete unknowns. The mesh is the set of locations where the discrete solution is computed. In order to use this scheme, the first and second order partial derivatives are replaced by their respective Taylors series finite difference approximations. Therefore, Equations (9) and (10) when expressed in finite difference form become.

$$
\begin{gathered}
\frac{U_{i}^{n+1}-U_{i}^{n}}{k}=\frac{U_{i+1}^{n}-2 U_{i}^{n}+U_{i-1}^{n}}{k^{2}}+v_{0} \frac{U_{i+1}^{n}-U_{i}^{n}}{k}+G r \theta_{i}^{n} \\
\frac{\theta_{i}^{n+1}-\theta_{i}^{n}}{k}=\frac{1}{p_{r}} \frac{\theta_{i+1}^{n}-2 \theta_{i}^{n}+\theta_{i-1}^{n}}{k^{2}}+E c\left(\frac{U_{i+1}^{n}-U_{i}^{n}}{k}\right)^{2}
\end{gathered}
$$

We used MATLAB to solve the explicit finite difference equations generated in Equations (12) and (13).

\section{Results and Discussions}

The solutions of the flow field presented by equations (12) and (13) are plotted for different values of Prandtl number, $P r$ and Grashoff number, $G r$ Eckert number, $E \mathcal{C}$ and Suction numbers $v_{0}$. In Figure 3, it has been depicted that as the Suction number increases the velocity profile decreases. This is because sucking decelerates fluid particles through the wall of the plate thereby reducing the growth of the boundary and thermal layer. It is also noted that an increase in Suction number cause a decrease in temperature distribution of the flow as shown in Figure 4. On the other hand, Figure 5 and Figure 6 depict the effect of Grash off number on both velocity and temperature. Grashoff number signifies the relative effect of buoyancy force on the boundary layer in comparison to viscous force. An increase in Grashoff number implies buoyancy force dominates 


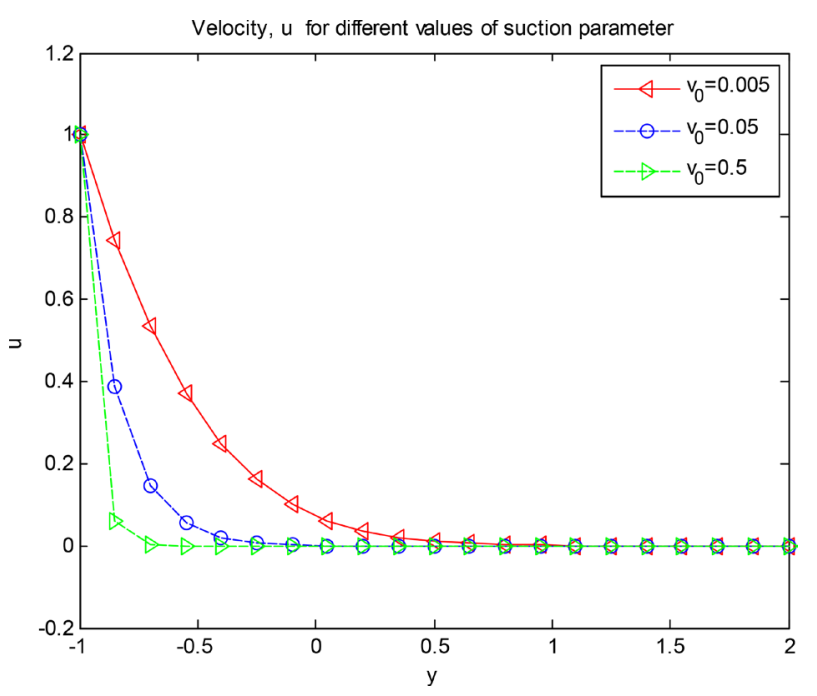

Figure 3. Effect of suction on velocity.

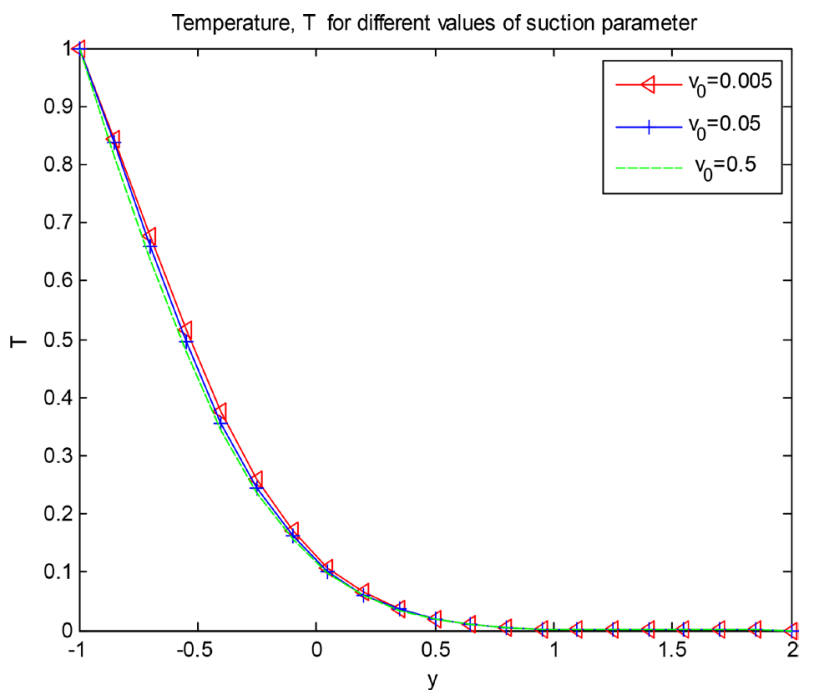

Figure 4. Effect of suction on temperature.

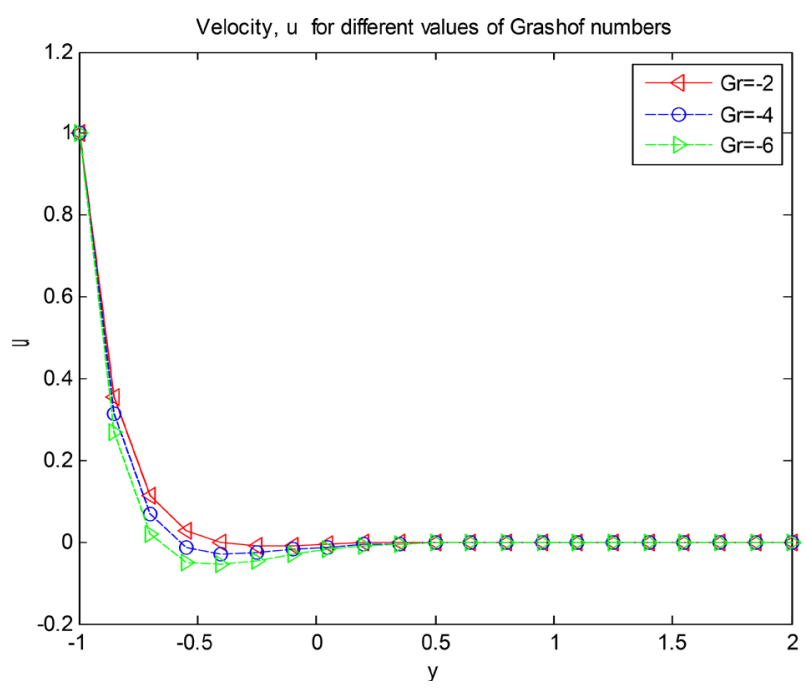

Figure 5. Effect of Grashof number on velocity. 
the viscous force. It was found to cause an increase in temperature of the flow field as indicated in Figure 6. However, in Figure 5, it was found that a rise in Grashof number caused a decrease in velocity profiles.

On the contribution of Eckert number to the flow field, it was found that an increase in Eckert number had a minimal effect on the velocity profiles as depicted in Figure 7 while in Figure 8, an increase in Eckert number led to a rise in the temperature distribution. The contribution of Prandtl number on temperature is shown in Figure 9. Prandtl number relates viscous force to thermal force. An increase in Prandtl numbers signifies a more viscous and heavier fluid. It was found that the temperature decreases drastically for the increase of Prandtl number as depicted by Figure 9 .

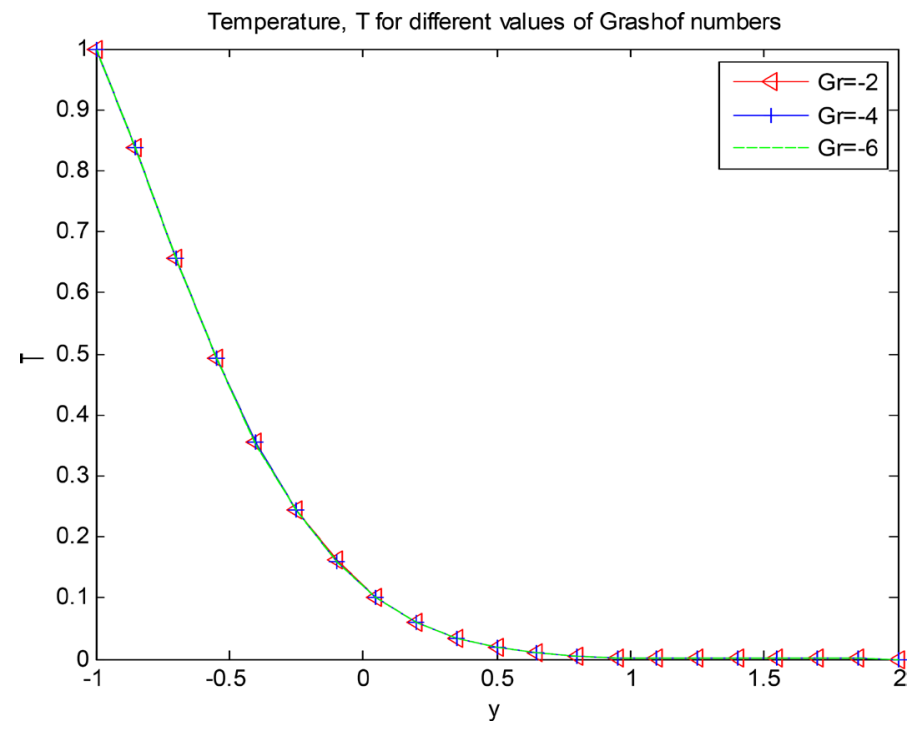

Figure 6. Effect of Grashof number on temperature.

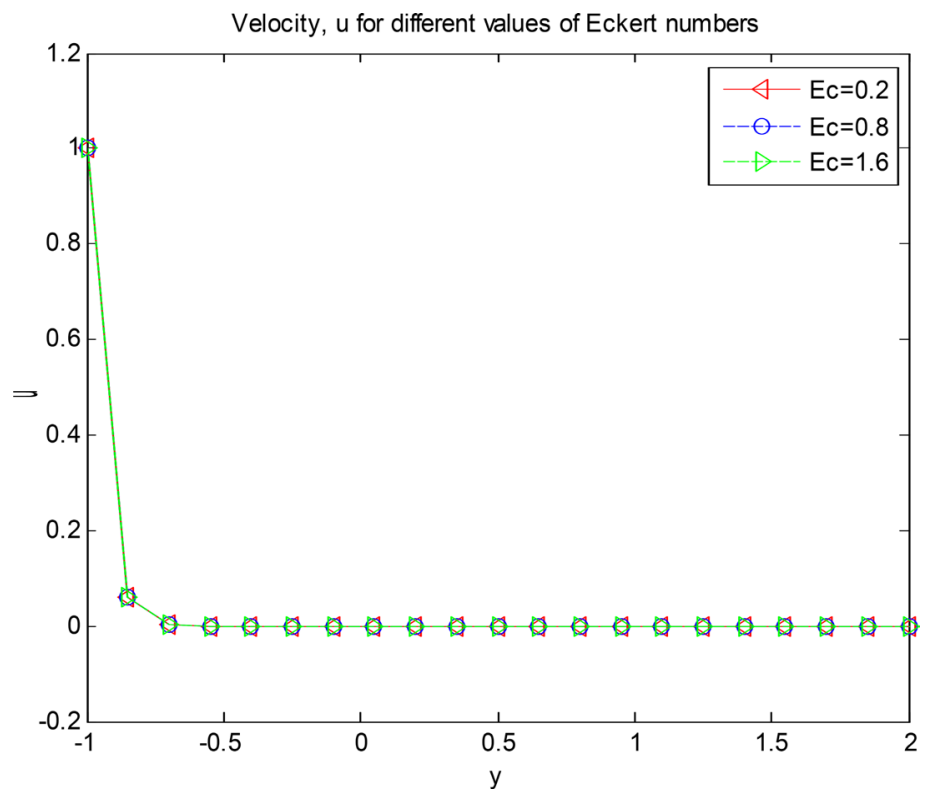

Figure 7. Effect of Eckert number on velocity. 


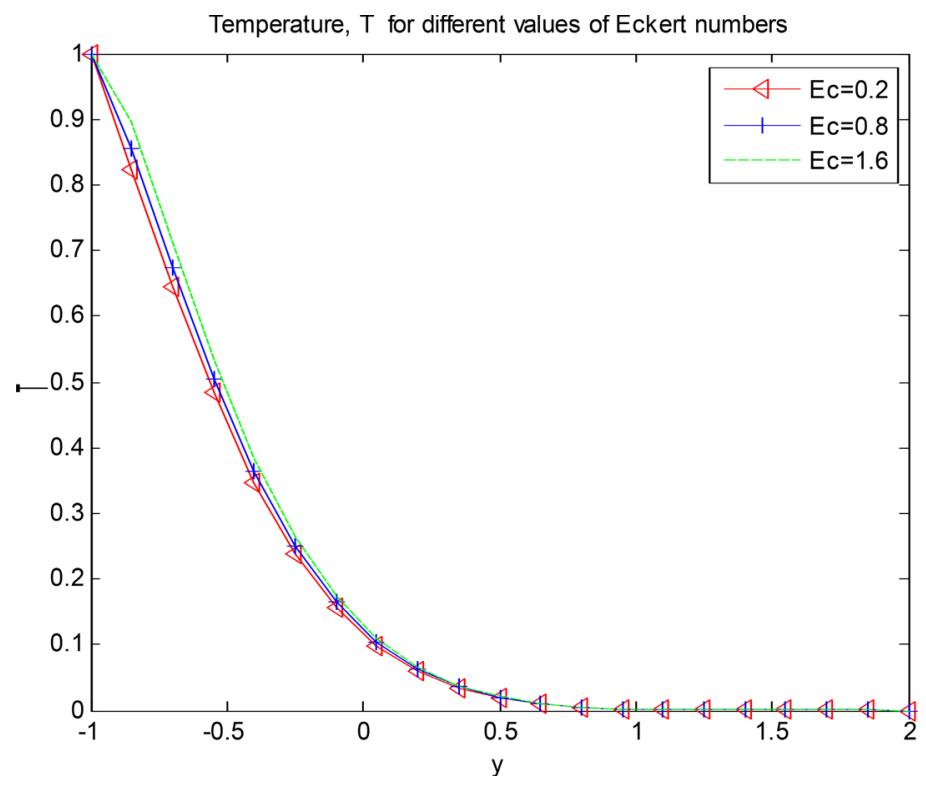

Figure 8. Effect of Eckert number on temperature.

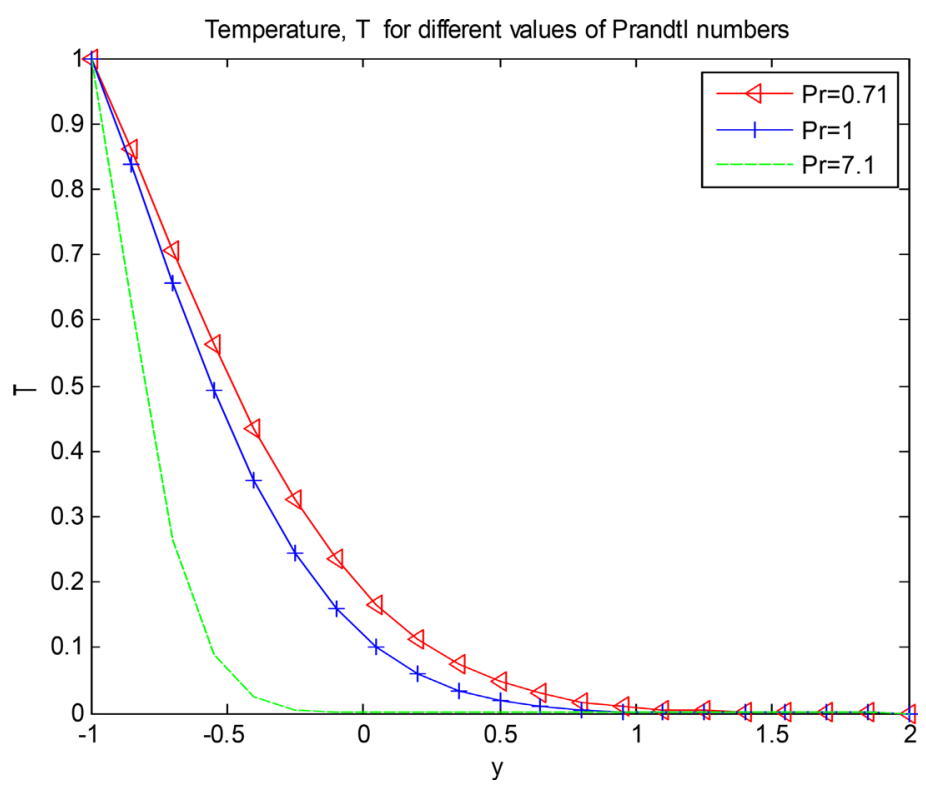

Figure 9. Effect of Prandtl number on temperature.

\section{Conclusion}

Velocity profiles and temperature distribution on an unsteady flow of an incompressible, viscous and electrically conducting fluid over a vertical plate in the presence of a uniform magnetic field have been investigated. Suction and Prandtl Numbers were found to have a great effect on velocity profiles and temperature distribution respectively. Results obtained for various values of these flow parameters have been found to suitably agree with the physical situation of the flow. It has been noted that an increase in Hartmann number causes a decrease in velocity profiles while an increase in Prandtl Number leads to a fall in temperature distribution. 


\section{Conflicts of Interest}

The authors declare no conflicts of interest regarding the publication of this paper.

\section{References}

[1] Adel, A.M., Soliman, R.K. and Ahemed, A.A. (2003) Similarity Analysis in Magneto Hydrodynamics: Hall Effects on Free Convection Flow and Mass Transfer past a Semi-Infinite Vertical Flat Plate. International Journal of Non-Linear Mechanics, 38, 513-520. https://doi.org/10.1016/S0020-7462(01)00077-4

[2] Alireza, R., Morteza, A., Imam, R., Bengt, S., Ganji, D.D. and Mehdi, G. (2018) Heat Transfer and MHD Flow of Non-Newtonian Maxwell Fluid through a Parallel Plate Channel. Analytical and Numerical Solution, Journal of Mechanical Science, 9, 61-70. https://doi.org/10.5194/ms-9-61-2018

[3] Amenya, O.R., Nyabuto, R. and Sigey, J.K. (2018) An Analysis of Unsteady MHD Fluid Flow in a Vertical Semi-Infinite Plate with Constant Transverse Magnetic Field. International Journal of Scientific Engineering and Research, 6, 98-102.

[4] Jagadeeswara, K., Vijaya, S. and Syam, M. (1987) MHD Free Convective Flow past a Hot Vertical Porous Plate. Defence Science Journal, 37, 327-332. https://doi.org/10.14429/dsj.37.5918

[5] Jabeen, K., Mushtaq, M. and Akram, R.M. (2020) Analysis of the MHD Boundary Layer Flow over a Nonlinear Stretching Sheet in a Porous Medium Using Semi Analytical Approaches. Mathematical Problems in Engineering, 2020, 1-9. https://doi.org/10.1155/2020/3012854

[6] Loganathan, P. and Sivapoornapriya, C. (2016) Unsteady Natural Convective Flow over an Impulsively Started Semi-Infinite Vertical Plate in the Presence of Porous Medium with Chemical Reaction. Journal of Applied Fluid Mechanics, 9, 95-102. https://doi.org/10.18869/acadpub.jafm.68.224.23951

[7] Mohammad, S.A. and Mohammad, A. (2017) Numerical Analysis of Two Dimensional MHD Free Convection and Mass Transfer of Fresh and Salt Water on an Inclined Plate with Hall Current and Constant Heat Flux. Athens Journal of Technology and Engineering, 125-149. https://doi.org/10.30958/ajte.4-2-4

[8] Muhammad, M.K., Muhammad, A.S. and Zahid, R. (2016) Magneto-Hydro Dynamic Generation. International Journal of Scientific and Engineering Research, 7, 281-289.

[9] Samwel, O.M., Obed, C.D., Emmanuel, U.A. and Jemima, N.O. (2012) Magneto Hydrodynamics Power Generation Using Salty Water. Asian Journal of Natural and Applied Sciences, 1, 1-4.

[10] Yahaya, S.D., Zainal, A.A., Zuhaila, I. and Faisal, S. (2018) Electrical Unsteady MHD Natural Convection Flow of Nano Fluid with Thermal Stratification and Heat Generation/Absorption. Mathematica, 34, 393-417. https://doi.org/10.11113/matematika.v34.n2.1016

[11] Kim, Y.J. (2000) Unsteady MHD Convective Heat Transfer past a Semi-Infinite Vertical Porous Moving Plate with Variable Suction. International Journal of Engineering Science, 38, 822-845. https://doi.org/10.1016/S0020-7225(99)00063-4

[12] Owuor, O.C., Kinyanjui, M.N. and Kiogora, P.R. (2020) Hydromagnetic Coquette Flow between Two Vertical Semi-Infinite Permeable Plates. International Journal of Advances in Applied Mathematics and Mechanics, 7, 1-13. 